\title{
A study for association and interaction analysis to metabolic syndrome and the ESR 1 gene on cardiovascular autonomic neuropathy in a Chinese Han population
}

Fangfang Zeng ${ }^{2}$, Linuo Zhou ${ }^{2}$ and Zihui Tang ${ }^{1 *}$

\begin{abstract}
Background: The aim of this study was to investigate the association and interaction of metabolic syndrome (MetS) and estrogen receptor alpha 1 (ESR1) gene polymorphisms on cardiovascular autonomic neuropathy (CAN).

Methods: A large-scale, population-based study was conducted to analyze the interaction of MetS and ESR1 gene polymorphisms to CAN, including a total of 1977 Chinese subjects. The most common studied single nucleotide polymorphism of ESR1 gene-rs9340799, was genotyped. Multiple logistic regression (MLR) was performed to evaluate the interaction effect of environmental variables and gene polymorphisms. Interaction on an additive scale can be calculated by using the relative excess risk due to interaction (RERI), the proportion attributable to interaction (AP), and the synergy index (S).
\end{abstract}

Results: After controlling potential confounders, MLR showed that significant association between MetS and CAN $(p<0.001)$. Interestingly, we found that the participants with MetS bearing the minor allele $G$ had an increased CAN prevalence comparing those with allele $\mathrm{A}(\mathrm{p}=0.045)$, and a positive interaction was estimated by using RETI = 0.396 (95\% Cl 0.262 to 0.598), AP = 0.216 (95\% Cl -0.784 to 1.216) and S = 1.906 (95 \% Cl 0.905 to 4.015).

Conclusion: The present findings suggest that MetS is significantly associated with CAN and provide evidence for the hypothesis that MetS and ESR1 gene polymorphism (rs9340799) have interactive effects on CAN.

ClinicalTrials gov Identifier NCT02461342

Keywords: Metabolic syndrome, Estrogen receptor alpha gene, Cardiovascular autonomic neuropathy, Interaction analysis

\section{Background}

The autonomic nervous system plays a critical role in regulating the function of numerous systems/organs, exerting its control through a broad network of afferent and efferent small nerve fibres. Cardiovascular autonomic neuropathy $(\mathrm{CAN})$ involves the autonomic nerve fibres innervating the heart and blood vessels [1], and is associated with a high risk of cardiac arrhythmias and sudden

\footnotetext{
*Correspondence: dr_zhtang@yeah.net

1 Department of Endocrinology and Metabolism, Shanghai Tongji Hospital, Tongji University School of Medicine, Shanghai 200065, China Full list of author information is available at the end of the article
}

death, possibly related to silent myocardial ischemia [2]. CAN is rapidly growing in all populations worldwide, particularly in the developing world, and is a prevalent form of diabetic autonomic neuropathy [3]. The reported prevalence of CAN varies from $2.5 \%$ to as high as $90 \%$, depending on the criteria used to identify CAN and the population studied [4].

The metabolic syndrome (MetS), made up of a cluster of cardiovascular risk factors including obesity, abdominal fat distribution, disorders of glucose and lipid metabolism and hypertension, increases the risk of future coronary and heart disease [5]. Hyperglycaemia and glycaemic variability play an important part in the 
development of autonomic neuropathy by contributing to oxidative stress that leads to neural damage [6]. Obesity and hypertension also contribute to cardiac autonomic dysfunction [7]. According to our previous study, MetS was strongly and independently associated with heart rate variability, and fasting plasma glucose and blood pressure were negatively correlated with cardiovascular autonomic function [8].

Oestrogen receptor $\alpha(E R \alpha)$ is encoded by the ESR1 gene which is located on the chromosome 6 (6q25.1). The beneficial effects of oestrogens on the cardiovascular system are mediated by the ER $\alpha$, which is also found in the autonomic centres of the brain stem involved in cardiovascular regulation, and oestrogen can access autonomic preganglionic cells in the central nervous system [9]. Part of the cardiovascular protective effect of oestrogen may relate to the beneficial effect of oestrogen on cardiac autonomic function. A variety of single nucleotide polymorphisms (SNPs) of the ESR1 gene are associated with cardiovascular disease. Of the polymorphisms identified in the ESR1 gene, rs9340799 is one of the most widely investigated SNPs [10]. The rs9340799 GG genotype is significantly associated with higher diastolic mean blood pressure and lower heart rate [11].

Environmental changes can modify the gene effect in human complex diseases, such as CAN. Our previous studies investigated the associations between MetS and CAN, and risk factors analysis for the outcome [8]. However, little was known about the relationships of MetS and ESR1 gene on CAN in Chinese Han population. In the present study, we hypothesize that MetS may modify the association of the ESR1 gene and CAN, meaning that MetS interacts with the ESR1 gene to increase susceptibility to CAN. The purpose of this study is to evaluate the extent to which MetS and the ESR1 gene are associated with CAN, and the impact of MetS interaction with the ESR1 gene on the outcome in Chinese Han population.

\section{Methods}

\section{Study population}

A risk factors survey for CAN was detailed earlier [12, 13], which was carried out in a random sample of the Chinese Han population. This study was approved by the Ethics Committee of Shanghai Tongji Hospital, Shanghai, China. Participants were recruited from rural and urban communities in Shanghai. Survey participants with undiagnosed CAN, aged $30-80$ years, were included in this study. Our study invited a total of 3012 subjects to a screening visit between 2011 and 2012. Written consent was obtained from all patients before the study. As we described earlier [12, 13], some subjects were excluded from the study to eliminate potential confounding factors that may have influenced their CA function. Briefly, the exclusion criteria were as follows: (1) history or findings of arrhythmia and hyperthyroidism or hypothyroidism; (2) pregnancy or lactation; (3) serious hepatic or renal dysfunctions (GFR $<30 \mathrm{~mL} / \mathrm{min} / 1.73 \mathrm{~m}^{2}$ ). Complete clinical baseline data were obtained for $2092(69.46 \%)$ of the participants.

\section{Measurement}

Participants were interviewed for the documentation of medical histories, medication, and history of smoking habits. Laboratory assessment for cardiovascular disease risk factors was completed, along with standardized examination for heart rate variability (HRV). As we mentioned earlier $[12,13]$, all study subjects underwent a complete clinical baseline characteristics evaluation after an 8-hour fast, which included: (1) history and physical examination; (2) heart rate and blood pressure; (3) fasting serum glucose and insulin; and (4) fasting plasma lipids. Systolic and diastolic blood pressure (BP) values were recorded as the mean of two physician-obtained measurements taken from the left arm of the seated participant. The day-to-day and inter-assay coefficients of variation at the central laboratory in our hospital for all analyses were between 1 and $3 \%$.

HTN was defined as BP $\geq 140 / 90 \mathrm{mmHg}$, or a history of hypertension medication. Body mass index (BMI) was calculated with weight in kilograms divided by the square of height in meters. DM was defined by oral glucose tolerance test (OGTT) or the use of insulin or hypoglycaemic medications. MetS was diagnosed according to the updated National Cholesterol Education Program/Adult Treatment Panel III criteria (WHO Western Pacific Region obesity criteria) in individuals meeting three or more of the criteria [14].

\section{SNP genotyping}

The genomic DNA was isolated from whole blood by proteinase $\mathrm{K}$ digestion followed by phenol-chloroform extraction. ESR1 (rs9340799) in 2092 Chinese Han participants was genotyped using iPLEX (Sequenom, San Diego, CA, USA) and detected by matrix-assisted laser desorption/ionization-time of flight mass spectrometry. Of these subjects, 1977 participants with complete clinical and genotype data were available for data analysis in this study. There was a $99.9 \%$ genotype concordance rate when duplicated samples were compared across plates.

\section{The study outcome}

In our large-scale, population-based study, this test was applied to evaluate CA function. HRV was measured non-invasively by power spectral analysis. As we described earlier, before CA function assessment, participants were to avoid alcohol, smoking, and coffee for 
$24 \mathrm{~h}$, in order to induce a calm and quiet state. Shortterm HRV analysis was performed for all subjects using a computer-aided examination and evaluation system for spectral analysis to investigate changes in autonomic regulation. In this study, CAN was diagnosed based on at least two abnormal cardiovascular autonomic reflex test results based on short-term HRV tests $[15,16]$.

\section{Statistical analysis}

Continuous variables were tested for normal distribution using the Kolmogorov-Smirnov test. Variables that were not normally distributed were log-transformed to approximate normal distribution for analysis. Results are described as mean \pm SD or median, unless stated otherwise. Differences in variables between subjects with non-MetS and MetS were determined by unpaired $t$ test. Between groups, differences in properties were accessed by $\chi^{2}$ analysis. Univariate logistic regression was performed to determine variables associated with CAN, and to estimate confounding factors possibly disturbing the relation of MetS and SNP to CAN. Multivariable logistic regression (MLR) was carried out to control potential confounders for determining the independent association of variables with CAN. For interaction analysis, MLR was conducted to include two main variables and their interaction item to evaluate the interaction effect. Odds ratios (OR) with $95 \%$ confidence intervals (CI) were calculated for the relative risk of MetS and SNP with CAN.

As we mentioned earlier [17], three parameters of RERI, AP, and $\mathrm{S}$ were used to estimate measures of interaction on an additive scale. The $95 \%$ CI for the three parameters were estimated as the 2.5th and 97.5th percentiles of the resulting bootstrap sampling distribution. Results were analysed using the Statistical Package for Social Sciences for Windows version 16.0 (SPSS, Chicago, IL, USA). Tests were two-sided and a p-value of $<0.05$ was considered significant. For interaction analysis, a p-value of $<0.10$ was also considered to be significant.

\section{Results}

\section{Characteristics of participants}

The baseline clinical characteristics of the 1977 participants are listed in Table 1 . There were 1310 females and 667 males (mean age, $60.50 \pm 8.71$ years) in the total sample. The prevalences of HTN, DM and CAN were significantly frequent in participants with MetS $(\mathrm{p}<0.001$ for all). In addition, there were significantly higher SBP, DBP, glucose profiles, lipids profiles levels and HR in participants with MetS as compared to participants without MetS, while there were lower HDL level and HRV index values in participants with MetS.

\section{Genotyping}

The minor allele (G) frequency of rs9340799 within the ESR1 gene was 19.97 and $19.08 \%$ in participants with MetS and participants without MetS, respectively. There were no significant differences in minor allele $(G)$ frequency of this SNP between the two groups $(p=0.495)$. The minor allele (G) frequency was $0.217,0.306$ and 0.196 in a sample of Utah residents with Northern and Western European ancestry (CEU), a sample of Han Chinese in Beijing (HCB) and the present study sample, respectively (Table 2 ). The genotype frequency was within the Hardy-Weinberg equilibrium ( $p>0.05)$. The primer for rs9340799 SNP is listed in Table 2.

\section{Univariate analysis for CAN}

Univariate logistic regression models were described earlier [12]. The models were developed to include age, gender, BMI, glucose profiles, lipids profiles, renal functions, medical history and rs9340799 SNP. Independent variables of age, BMI, SBP, DBP, FPG, PBG, FINS, TG, HTN, $\mathrm{DM}$ and MetS were significantly associated with CAN ( $\mathrm{p}<0.05$ for all). Univariate logistic regression analysis showed MetS to be significantly associated with the outcome ( $\mathrm{p}<0.001)$; however, no significant association of rs9340799 SNP with CAN was reported $(\mathrm{p}=0.627)$.

\section{Multiple variables analysis for CAN}

Multiple variable logistic regression to include MetS and rs9340799 SNP, controlling for potential confounding factors of age, gender, PBG, FINS and renal functions, showed significant associations between MetS and CAN ( $p<0.001$, OR $=1.802$ and $95 \%$ CI 1.521-2.136, Table 3). There was also no significant association between rs9340799 SNP and CAN in MLR, controlling for potential confounding factors $(\mathrm{p}=0.893)$.

\section{Interaction of MetS and SNP for CAN}

MLR models were developed to include the two main effect variables of MetS and rs9340799 SNP, and its interaction of MetS with rs9340799. The interaction item between them was detected in the MLR model after adjustment for relevant potential confounders involving age, gender, PBG, FINS and renal functions $(\mathrm{p}=0.062$, Table 4 and Fig. 1). In participants without MetS, the CAN prevalence was similar between subjects with minor allele $\mathrm{G}$ and subjects with allele A (13.39 vs. $15.60 \%, \mathrm{p}=0.151$ ). However, in participants with MetS, the CAN prevalence was significantly frequent in subjects with minor allele $\mathrm{G}$ as compared with subjects with allele A (25.59 vs. $21.89 \% \mathrm{p}=0.045)$. The interaction effect was estimated as $\left(O R_{\text {Int }}=1.505,95 \%\right.$ CI 0.980 2.312). The interaction on an additive scale was estimated 
Table 1 The clinical baseline characteristics of individuals

\begin{tabular}{|c|c|c|c|c|}
\hline Variable & non-MetS & MetS & Entire sample & p value* \\
\hline \multicolumn{5}{|c|}{ Demographical information } \\
\hline $\mathrm{N}$ & 1194 & 783 & 1977 & \\
\hline Age years & $59.8 \pm 8.85$ & $61.56 \pm 8.37$ & $60.5 \pm 8.71$ & $<0.001$ \\
\hline Gender male, $\%$ & 409 (34.25\%) & 258 (32.95 \%) & 667 (33.74 \%) & 0.396 \\
\hline Height $\mathrm{cm}$ & $161.5 \pm 7.89$ & $161.48 \pm 7.79$ & $161.49 \pm 7.85$ & 0.942 \\
\hline Weight kg & $60.41 \pm 9.78$ & $67.69 \pm 10.5$ & $63.3 \pm 10.68$ & $<0.001$ \\
\hline $\mathrm{SBP} \mathrm{mmHg}$ & $122.61 \pm 17.33$ & $135.52 \pm 18.34$ & $127.71 \pm 18.82$ & $<0.001$ \\
\hline DBP $\mathrm{mmHg}$ & $77.82 \pm 9.38$ & $82.8 \pm 9.38$ & $79.79 \pm 9.69$ & $<0.001$ \\
\hline \multicolumn{5}{|l|}{ Medical history, \% } \\
\hline Smoking yes & $182(15.24 \%)$ & $122(15.58 \%)$ & 304 (15.38 \%) & 0.773 \\
\hline HTN yes & 371 (31.07\%) & 557 (71.14 \%) & 928 (46.94 \%) & $<0.001$ \\
\hline DM yes & $112(9.39 \%)$ & 307 (39.21\%) & $419(21.2 \%)$ & $<0.001$ \\
\hline CAN yes & $173(14.49 \%)$ & $190(24.27 \%)$ & $363(18.36 \%)$ & $<0.001$ \\
\hline \multicolumn{5}{|l|}{ Laboratory assays } \\
\hline FPG mmol/L & $5.01 \pm 1.27$ & $6.19 \pm 2.16$ & $5.48 \pm 1.78$ & $<0.001$ \\
\hline PBG mmol/L & $6.63 \pm 2.87$ & $9.08 \pm 4.02$ & $7.6 \pm 3.58$ & $<0.001$ \\
\hline FINS uml/L & $6.07 \pm 10.53$ & $9.07 \pm 14.08$ & $7.26 \pm 12.16$ & $<0.001$ \\
\hline $\mathrm{TC} \mathrm{mmol} / \mathrm{L}$ & $5.31 \pm 0.96$ & $5.33 \pm 1.07$ & $5.32 \pm 1$ & 0.603 \\
\hline $\mathrm{TG} \mathrm{mmol} / \mathrm{L}$ & $1.35 \pm 0.66$ & $2.26 \pm 1.14$ & $1.71 \pm 0.99$ & $<0.001$ \\
\hline $\mathrm{HDL} \mathrm{mmol} / \mathrm{L}$ & $1.46 \pm 0.32$ & $1.2 \pm 0.26$ & $1.36 \pm 0.33$ & $<0.001$ \\
\hline LDL mmol/L & $3.18 \pm 0.76$ & $3.21 \pm 0.8$ & $3.19 \pm 0.78$ & 0.212 \\
\hline $\mathrm{SCr} \mu \mathrm{mol} / \mathrm{L}$ & $76.56 \pm 28.72$ & $79.14 \pm 22.9$ & $77.58 \pm 26.59$ & 0.003 \\
\hline $\mathrm{UA} \mu \mathrm{mol} / \mathrm{L}$ & $265.96 \pm 78.37$ & $302.66 \pm 87.82$ & $280.51 \pm 84.17$ & $<0.001$ \\
\hline \multicolumn{5}{|l|}{ HRV indices } \\
\hline HR bpm & $71.57 \pm 9.92$ & $73.96 \pm 10.34$ & $72.52 \pm 10.16$ & $<0.001$ \\
\hline $\mathrm{TP} \mathrm{ms}^{2}$ & $938.73 \pm 728.92$ & $788.78 \pm 668.07$ & $879.34 \pm 709.16$ & $<0.001$ \\
\hline $\mathrm{LF} m s^{2}$ & $210.37 \pm 219.54$ & $164.72 \pm 189.4$ & $192.29 \pm 209.3$ & $<0.001$ \\
\hline $\mathrm{HF} \mathrm{ms}^{2}$ & $203.82 \pm 241.68$ & $155.52 \pm 183.24$ & $184.69 \pm 221.63$ & $<0.001$ \\
\hline $\mathrm{LF} / \mathrm{HF}$ & $1.67 \pm 2.05$ & $1.74 \pm 1.87$ & $1.7 \pm 1.98$ & 0.307 \\
\hline \multicolumn{5}{|l|}{ SNP } \\
\hline rs9340799 & 237 (19.97 \%) & 147 (19.08\%) & 384 (19.62 \%) & 0.495 \\
\hline
\end{tabular}

* Difference analysis between non-MetS and MetS groups

SBP systolic blood pressure, DBP diastolic blood pressure, FPG fasting plasma glucose, PBG plasma blood glucose, FINS fasting blood insulin, TC serum total cholesterol, $T G$ triglyceride, UA uric acid, $H D L$ high-density lipoprotein cholesterol, $L D L$ low density lipoprotein cholesterol, $S C r$ serum creatinine, $U A$ uric acid, $H R$ heart rate, $T P$ total power of variance, LF low frequency, HF high frequency, HTN hypertension, MetS metabolic syndrome, DM diabetes mellitus, CAN cardiovascular autonomic neuropathy, SNP single nucleotide polymorphism

Table 2 Information of single nucleotide polymorphism of ESR1 and its primer

\begin{tabular}{lllllll}
\hline SNP & Gene & CH & Allele & MAF $^{\text {CEU }}$ & MAF HCB $^{\text {HAF }}$ & MAF $^{\text {TPS }}$ \\
\hline rs9340799 & ESR1 & 6 & G/A & 0.217 & 0.306 & 0.196 \\
Up-stream & CATCTGAGTTCCAAATGTCC & & & & \\
Down-stream & GGATGAGCATTGGTCTCTAA & & & &
\end{tabular}

SNP single nucleotide polymorphism, $C H$ chromosome, $M A F^{C E U}$ minor allele frequency in Utah residents with Northern and Western European ancestry sample, $M A F^{H C B}$ minor allele frequency in Han Chinese in Beijing sample, $M A F^{T P S}$ minor allele frequency in the present sample, $H W E$ p value from Hardy-Weinberg Equilibrium test in our sample 
Table 3 Multiple variables analysis for metabolic syndrome and rs9340799 for cardiovascular autonomic neuropathy

\begin{tabular}{lrrrrr}
\hline Variables & \multicolumn{1}{l}{$\boldsymbol{\beta}$} & S.E. & p value & OR & $\mathbf{9 5 . 0} \% \mathbf{C l}$ \\
\hline MetS & 0.589 & 0.087 & $<0.001$ & 1.802 & $1.521-2.136$ \\
rs9340799 & -0.114 & 0.109 & 0.299 & 0.893 & $0.720-1.106$ \\
\hline
\end{tabular}

MetS metabolic syndrome, $B M I$ body mass index, and multiple variables logistic model adjusted for age, gender, PBG, FINS and renal functions

Table 4 The interaction analysis of MetS and rs9340799 for CAN

\begin{tabular}{lrllll}
\hline Variables & $\boldsymbol{\beta}$ & S.E. & $\mathbf{p}$ value & OR & $\mathbf{9 5 . 0} \% \mathbf{C l}$ \\
\hline MetS & 0.551 & 0.092 & 0.001 & 1.735 & $1.449-2.079$ \\
rs9340799 & -0.354 & 0.259 & 0.226 & 0.702 & $0.314-1.159$ \\
MetS by rs9340799 & 0.409 & 0.219 & 0.062 & 1.505 & $0.980-2.312$ \\
RERI & 0.396 & & & & $0.262-0.598$ \\
AP & 0.216 & & & & $-0.784-1.216$ \\
S & 1.906 & & & & $0.905-4.015$ \\
\hline
\end{tabular}

MetS metabolic syndrome, $R E R /$ relative excess risk due to interaction, $A P$ proportion attributable to interaction, $S$ synergy index; and multiple variables logistic model adjusted for age, gender, PBG, FINS and renal functions

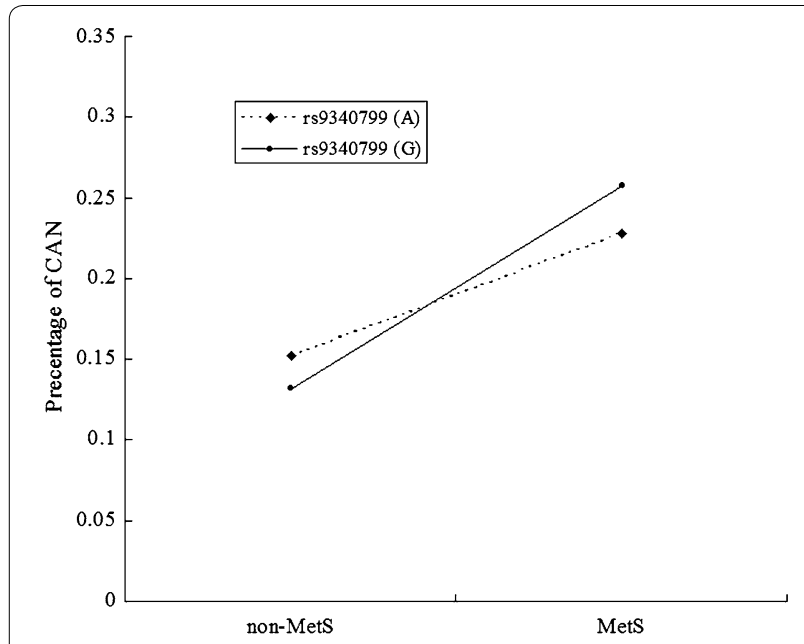

Fig. 1 Interaction analysis of ESR1 (rs9340799) and metabolic syndrome for CAN. Interaction of MetS and rs9340799 (MetS*rs9340799 ORGEI $=1.505,95 \% \mathrm{Cl} 0.98-2.312, \mathrm{p}=0.062)$

as $(\mathrm{RERI}=0.396,95 \% \mathrm{CI} 0.262-0.598 ; \mathrm{AP}=0.216,95 \%$ CI -0.784 to 1.216 ; $\mathrm{S}=1.906,95 \%$ CI $0.905-4.015)$.

\section{Discussion}

We conducted a large-scale study to evaluate the interaction effect of MetS and the ESR1 gene on CAN in a sample of the Chinese population. To our knowledge, this is the first study to perform a synergistic analysis of
MetS and rs9340799 polymorphism of the ESR1 gene on $\mathrm{CAN}$ in the general Chinese population. Moreover, it is very important for us to understand environmental and genetic influences on diseases. Since more than one risk factor contributes to the progression and deterioration of a disease, finding one risk factor which is controllable, especially among modifiable risk factors, would be of great value to physicians and patients.

In the present study, there was no significant difference of allele frequency of rs9340799 between participants with and without MetS. No association of rs9340799 with CAN was detected. Univariate and multiple variable analysis provided evidence that MetS was strongly and independently associated with CAN ( $\mathrm{p}<0.001$, respectively), and the results were consistent with previous studies that MetS was negatively correlated with HRV and cardiovascular autonomic function [18].

Because the incidence of coronary heart disease (CHD) rises significantly after menopause, it has been hypothesized that women's CHD advantage before menopause, in comparison with men of the same age, could be due to the protective effects of oestrogens [19]. Additionally, oestrogen treatment, long-term or short-term, has been shown to reduce sympathetic drive and improve cardiac autonomic control [20], the same as with changes in HRV across the menstrual cycle [21]. The cardiac autonomic protective effect of oestrogen may be a partial factor in the undefined physiological mechanisms of beneficial effects of oestrogen on cardiovascular disease.

Previous studies have suggested that polymorphism rs9340799, one of the widely identified polymorphisms of ESR1, may relate to ischemic heart disease [22, 23]. As for the association of polymorphism rs9340799 with cardiovascular autonomic function, Matsunaga and his colleagues recruited 252 young healthy males to examine the association of ESR1 polymorphism rs9340799 with short-term HRV [11], and found that rs9340799 GG genotype was significantly associated with higher diastolic and mean blood pressure, but lower heart rate. In our present study, no association was found between polymorphism rs9340799 and HRV indices (data not shown).

Our important finding was that a significant interaction of MetS and rs9340799 is associated with CAN in the Chinese population. In the MLR analysis, the interaction term was indicated as a significant positive interaction of the two main factors that affect CAN ( $\mathrm{p}=0.062$, RETI $>0, A P>0, S>1$ ), suggesting that the combined effect of MetS and rs9340799 on CAN was greater than the sum of the individual effects of the two factors. It is noteworthy that MetS individuals bearing the minor $\mathrm{G}$ allele were more susceptible to the progression of CAN. To our knowledge, this is the first analysis of the interaction of MetS and ESR1 variant on CAN susceptibility. 
Our findings suggested that MetS interact with ESR1 polymorphisms to affect progression of CAN. In clinical practice, we may predict MetS patients with ESR1 polymorphisms more susceptibility to associate with CAN. The analysis for ESR1 polymorphisms and MetS to predict progression of CAN will be perform in followup studies. However, the underlying mechanism is still unknown. In the insulin-resistance atherosclerosis family study, rs9340799 was found to be positively associated with MetS [24], implying that ESR1 (rs9340799) may disturb CAN susceptibility through unknown mechanisms contributing to MetS, which is independently and significantly associated with CAN [25]. Additionally, the indices of MetS such blood pressure and glucose profiles might modify effects on the interaction, or separately associate with the polymorphism.

Adipocyte hyperplasia and hypertrophy, insulin resistance and glucose intolerance were associated with ER $\alpha$ absence in the knock-out mice model, suggesting that the oestrogen/ER $\alpha$ signalling pathway is critical in adipose tissue and may be involved in a mechanism of energy metabolism [26]. A human male with an ESR1-null mutation had insulin resistance, impaired glucose tolerance, obesity and increased height [27]. The rs9340799 mutation was associated with body mass index in an ageadjusted case-control study [28], and the rs9340799 AA genotype was associated with lower body mass index and waist in middle-aged women [29]. In contrast, the rs9340799 GG genotype was associated with significantly higher serum total cholesterol and LDL cholesterol levels compared to those with the AA or AG genotype [24]. No association was found between the genotype of rs9340799 and lipid profile in our present study (data not shown). Whether polymorphism rs9340799 of the ESR1 gene could influence serum lipid levels and the underlying molecular mechanisms still needs to be determined.

Several limitations of this study warrant comment. This study was based on a cross-sectional study; synergistic effects analysis requires a larger sample size, covering age groups other than 30-80 years and more geographic representations of China. The synergistic results in the present study need to be verified by future follow-up studies. It is also important that the study was performed on Chinese individuals and our findings may not be relevant to people of other ethnicities.

In conclusion, our findings indicate that MetS is significantly associated with CAN, and we offer evidence to support the hypothesis that MetS and ESR1 gene polymorphism (rs9340799) have positive interactive effects on CAN. Abnormal metabolic status may modify the effect of the ESR1 gene on the progression of CAN. This indicates that metabolic improvement of genetically high-risk individuals could attenuate the risk and progression of CAN.

\section{Authors' contributions}

FZ carried out the molecular genetic studies, participated in the sequence alignment and drafted the manuscript. LZ participated in the design of the study and performed the statistical analysis. ZT conceived of the study, and participated in its design and coordination and helped to draft the manuscript. All authors read and approved the final manuscript.

\section{Author details}

${ }^{1}$ Department of Endocrinology and Metabolism, Shanghai Tongji Hospital, Tongji University School of Medicine, Shanghai 200065, China. ${ }^{2}$ Department of Endocrinology and Metabolism, Fudan University Huashan Hospital, Shanghai, China.

\section{Acknowledgements \\ We thank the grant from Shanghai Tongji Hospital to support the study.}

\section{Competing interests}

The authors declare that they have no competing interests.

\section{Ethical approval}

All procedures performed in studies involving human participants were in accordance with the ethical standards of the institutional and/or national research committee and with the 1964 Helsinki declaration and its later amendments or comparable ethical standards.

\section{Funding sources}

Grants from the Clinical Medicine Foundation of Shanghai Tongji Hospital.

Informed consent

Informed consent was obtained from all individual participants included in the study.

Received: 13 March 2016 Accepted: 10 July 2016

Published online: 22 July 2016

References

1. Vinik Al, Ziegler D. Diabetic cardiovascular autonomic neuropathy. Circulation. 2007;115:387-97.

2. Pop-Busui R. Cardiac autonomic neuropathy in diabetes: a clinical perspective. Diabetes Care. 2010;33:434-41.

3. Hazari MA, Khan RT, Reddy BR, Hassan MA. Cardiovascular autonomic dysfunction in type 2 diabetes mellitus and essential hypertension in a South Indian population. Neurosciences. 2012;17:173-5.

4. The effect of intensive diabetes therapy on measures of autonomic nervous system function in the Diabetes Control and Complications Trial (DCCT). Diabetologia. 1998:41 (4):416-23.

5. Lakka HM, Laaksonen DE, Lakka TA, Niskanen LK, Kumpusalo E, et al. The metabolic syndrome and total and cardiovascular disease mortality in middle-aged men. JAMA. 2002;288:2709-16.

6. Fleischer J. Diabetic autonomic imbalance and glycemic variability. J Diabetes Sci Technol. 2012:6:1207-15.

7. Ziegler D, Zentai C, Perz S, Rathmann W, Haastert B, et al. Selective contribution of diabetes and other cardiovascular risk factors to cardiac autonomic dysfunction in the general population. Exp Clin Endocrinol Diabetes. 2006;114:153-9.

8. Tang ZH, Liu J, Zeng F, Li Z, Yu X, et al. Comparison of prediction model for cardiovascular autonomic dysfunction using artificial neural network and logistic regression analysis. PLoS One. 2013;8:e70571.

9. Saleh TM, Connell BJ. Role of oestrogen in the central regulation of autonomic function. Clin Exp Pharmacol Physiol. 2007;34:827-32.

10. Casazza K, Page GP, Fernandez JR. The association between the rs2234693 and rs9340799 estrogen receptor alpha gene polymorphisms and risk factors for cardiovascular disease: a review. Biol Res Nurs. 2010;12:84-97.

11. Matsunaga T, Gu N, Yamazaki H, Adachi T, Yasuda K, et al. Association of estrogen receptor-alpha gene polymorphisms with cardiac autonomic nervous activity in healthy young Japanese males. Clin Chim Acta. 2010:411:505-9. 
12. Liu J, Tang ZH, Zeng F, Li Z, Zhou L. Artificial neural network models for prediction of cardiovascular autonomic dysfunction in general Chinese population. BMC Med Inform Decis Mak. 2013;13:80,

13. Li Z, Tang ZH, Zeng F, Zhou L. Associations between the severity of metabolic syndrome and cardiovascular autonomic function in a Chinese population. J Endocrinol Invest. 2013;36:993-9.

14. Grundy SM, Hansen B, Smith SC Jr, Cleeman JI, Kahn RA. Clinical management of metabolic syndrome: report of the American Heart Association/ National Heart, Lung, and Blood Institute/American Diabetes Association conference on scientific issues related to management. Circulation. 2004:109:551-6.

15. Zeng F, Tang ZH, Li Z, Yu X, Zhou L. Normative reference of short-term heart rate variability and estimation of cardiovascular autonomic neuropathy prevalence in Chinese people. J Endocrinol Invest. 2014;37:385-91.

16. Tang ZH, Zeng F, Yu X, Zhou L. Bayesian estimation of cardiovascular autonomic neuropathy diagnostic test based on baroreflex sensitivity in the absence of a gold standard. Int J Cardiol. 2014;171:e78-80.

17. Liao XP, Zhu HW, Zeng F, Tang ZH. The association and interaction analysis of hypertension and uric acid on cardiovascular autonomic neuropathy. J Endocrinol Invest. 2015:38:1075-82.

18. Jarczok MN, Li J, Mauss D, Fischer JE, Thayer JF. Heart rate variability is associated with glycemic status after controlling for components of the metabolic syndrome. Int J Cardiol. 2013;167:855-61.

19. Lerner DJ, Kannel WB. Patterns of coronary heart disease morbidity and mortality in the sexes: a 26-year follow-up of the Framingham population. Am Heart J. 1986;111:383-90.

20. Gokce M, Karahan B, Yilmaz R, Orem C, Erdol C, et al. Long term effects of hormone replacement therapy on heart rate variability, QT interval, QT dispersion and frequencies of arrhythmia. Int J Cardiol. 2005;99:373-9.
21. Tenan MS, Brothers RM, Tweedell AJ, Hackney AC, Griffin L. Changes in resting heart rate variability across the menstrual cycle. Psychophysiology. 2014;51:996-1004.

22. Schuit SC, Oei HH, Witteman JC, Geurts van Kessel CH, van Meurs JB, et al. Estrogen receptor alpha gene polymorphisms and risk of myocardial infarction. JAMA. 2004;291:2969-77.

23. Rokach A, Pollak A, Rosen L, Friedlander Y, Blumenfeld A, et al. Estrogen receptor alpha gene polymorphisms are associated with the angiographic extent of coronary artery disease. J Clin Endocrinol Metab. 2005;90:6556-60

24. Molvarec A, Nagy B, Kovacs M, Walentin S, Imreh E, et al. Lipid, haemostatic and inflammatory variables in relation to the estrogen receptor alpha (ESR1) Pvull and Xbal gene polymorphisms. Clin Chim Acta. 2007;380:157-64

25. Soares-Miranda L, Sandercock G, Vale S, Santos R, Abreu S, et al. Metabolic syndrome, physical activity and cardiac autonomic function. Diabetes Metab Res Rev. 2012;28:363-9.

26. Heine PA, Taylor JA, Iwamoto GA, Lubahn DB, Cooke PS. Increased adipose tissue in male and female estrogen receptor-alpha knockout mice. Proc Natl Acad Sci USA. 2000;97:12729-34.

27. Smith EP, Boyd J, Frank GR, Takahashi H, Cohen RM, et al. Estrogen resistance caused by a mutation in the estrogen-receptor gene in a man. N Engl J Medicine. 1994;331:1056-61.

28. Mansur Ade P, Nogueira CC, Strunz CM, Aldrighi JM, Ramires JA. Genetic polymorphisms of estrogen receptors in patients with premature coronary artery disease. Arch Med Res. 2005;36:511-7.

29. Okura T, Koda M, Ando F, Niino N, Ohta S, et al. Association of polymorphisms in the estrogen receptor alpha gene with body fat distribution. Int J Obes Relat Metab Disord. 2003:27:1020-7.

\section{Submit your next manuscript to BioMed Central and we will help you at every step:}

- We accept pre-submission inquiries

- Our selector tool helps you to find the most relevant journal

- We provide round the clock customer support

- Convenient online submission

- Thorough peer review

- Inclusion in PubMed and all major indexing services

- Maximum visibility for your research

Submit your manuscript at www.biomedcentral.com/submit
O Biomed Central 